\title{
A Side-Fed Circularly-Polarized Patch Antenna with a Dielectric Loading
}

\author{
Ji-Young Jeong $^{1} \cdot$ Seung-Mo Choi ${ }^{2} \cdot$ Bayanmunkh Enkhbayar ${ }^{1} \cdot$ \\ Ononchimeg Sodnomtseren ${ }^{1} \cdot$ Bierng-Chearl Ahn ${ }^{1}$
}

\begin{abstract}
In this paper, we present the design and measurement of a side-fed circularly-polarized patch antenna with a dielectric loading. The antenna consists of a corner-truncated rectangular patch, an L-shaped ground plane, a dielectric loading material, and a coaxial probe. An antenna operating at the UHF band (910 MHz) for the RFID reader applications is optimized using a commercial software. The size of the patch is reduced by a factor of 1.73 by loading the patch with mono-cast(MC) nylon. Measurements of the fabricated antenna show performance characteristics comparable to those of much larger commercial RFID reader antennas.
\end{abstract}

Key words : Dielectric-Loaded Antenna, Circularly-Polarized Patch Antenna.

\section{Introduction}

Recently there have been intensive research and development efforts in RFID technologies ${ }^{[1]-[3]}$. As one of important components in the RFID system, the RFID reader antenna has been investigated by numerous researchers ${ }^{[4]-[7]}$. The tag read range is proportional to the square root of the reader antenna gain ${ }^{[8]}$. The reader antenna should have a gain large enough to ensure the reliable range performance.

In 2003, Chang and co-workers ${ }^{[9]}$ proposed a wideband patch antenna consisting of a corner-truncated rectangular patch, an air dielectric, an L-shaped ground plane and a coaxial probe horizontally feeding the patch on the patch edge through a vertical ground plane. They were able to increase the bandwidth of the patch by increasing the distance from the ground plane to the patch and by feeding the patch from side, not from bottom. With their feeding method, the probe length can be made small and the probe inductance is minimized, which results in wideband performance.

Reduction of the RFID reader antenna size is preferable for the sake of space economy. In this paper, we present the design and measurement of a circularly polarized patch antenna miniaturized by dielectric loading. The basic form of the antenna is same as the one proposed by Chang and co-workers ${ }^{[9]}$. A low-cost industrial dielectric material is used to load the patch and thus to reduce the patch size. Microwave Studio(MWS) ${ }^{\mathbb{B}}$, a widely-used commercial electromagnetic software, is used to obtain an optimum design of the antenna. Parametric studies are carried out for the antenna structure, from which we derive an optimum design. The designed antenna is fabricated and its performances are measured and compared with the simulation.

\section{Il . Antenna Design}

Fig. 1 shows the structure of the miniaturized circularlypolarized patch antenna. To reduce the patch size, the space between the ground plane and the patch is filled with a dielectric material. The antenna is analyzed and optimized using $\mathrm{MWS}^{\circledR}$. Initial design parameters are determined as follows.

As the first step in the design, a suitable dielectric loading material should be chosen. Ceramic materials are not suitable for this application due to its high cost and limited availability although they have higher permittivity. Industrial plastic materials are good choice because they are cheap and readily available. Next we calculate the patch size using Eqs. (1) and (2).

$$
\begin{aligned}
& L=\frac{\lambda}{2}-2 \Delta L \\
& \Delta L=0.412 h \frac{\left(\varepsilon_{r, e f f}+0.3\right)(W / h+0.264)}{\left(\varepsilon_{r, e f f}+0.258\right)(W / h+0.8)}
\end{aligned}
$$

where $\varepsilon_{r, e f f} 1$ is the effective dielectric constant of a transmission line of same width as the patch, $\lambda=c / f, W$ is the patch width, and $h\left(=h_{1}\right)$ is the distance from the ground plane to the patch. The ground-to-patch spacing $h_{1}$ is set at about $1 / 4$ of the patch size. For simplicity, the patch width $W_{3}$ is set to be equal to the patch length $L_{3}$.

Two diagonal corners of the patch is truncated in

Manuscript received September 6, 2007 ; October 2, 2007. (ID No. 20070906-021J)

${ }^{1}$ School of Electrical and Computer Engineering, Chungbuk University, Cheongju, Korea.

${ }^{2}$ Mito-RF Co., Cheongju, Korea. 


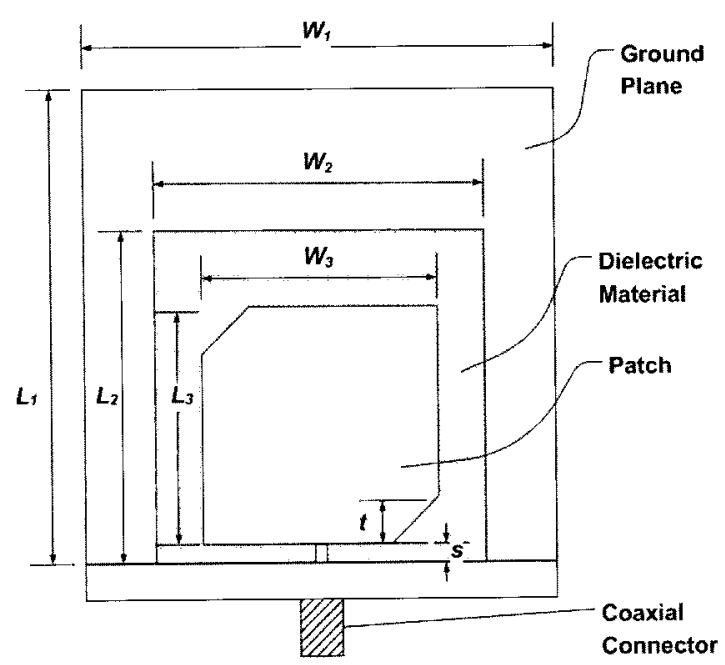

(a) Top view

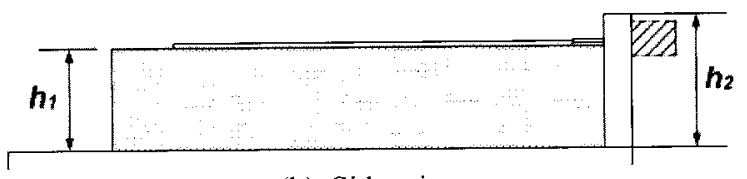

(b) Side view

Fig. 1. Structure of the proposed antenna.

order to obtain circularly-polarized radiation. The truncation of the patch modifies the resonant frequency according to Eq. (3) ${ }^{[10]}$.

$$
f_{r}^{\prime}=\frac{f_{r}}{\left(1-\frac{2 \Delta S}{S}\right)}
$$

Good circular-polarization performances are obtained with the truncation ratio $t / L_{3}$ around 0.2 . The truncation ratio is obviously dependent upon the ground-to-patch spacing $h_{1}$.

For minimal perturbations on the patch performance, sizes of the dielectric material and the ground plane should be at least 1.3 and 1.7 times the patch size. The patch is fed by a thin coaxial probe such as the center conductor of the SMA connector with a $1.42-\mathrm{mm}$ thick inner conductor and a $4.20-\mathrm{mm}$ thick outer conductor. The height $h_{2}$ of the vertical ground should be as small as possible in order not to disturb the radiation by the patch. In fact it should be same as the spacing $h_{1}$ between the horizontal ground plane and the patch. It is increased beyond $h_{1}$ only to accommodate the coaxial connector. Finally thicknesses of the ground plane and the patch are set at a convenient value such as $0.2 \mathrm{~mm}$ and $3 \mathrm{~mm}$, respectively, considering conducting materials to be used in the fabrication.

With initial parameters analytically obtained, we analyze the entire antenna structure using $\mathrm{MWS}^{\mathrm{R}}$ to study the dependence of the patch performance on various para-
Table 1. Patch size and gain versus the relative permittivity of the dielectric material.

$\left(f_{r}=910 \mathrm{MHz}, W_{1}=L_{1}=160, W_{2}=L_{2}=120, t=21, \quad s=7, h_{1}=20\right.$, $h_{2}=30$, unit: $\mathrm{mm}$ )

\begin{tabular}{|c|c|c|}
\hline $\begin{array}{c}\text { Relative } \\
\text { permittivity }\end{array}$ & $\begin{array}{c}\text { Patch size } \\
\left(W_{3}=L_{3}\right)\end{array}$ & $\begin{array}{c}\text { Gain } \\
(\mathrm{dBi})\end{array}$ \\
\hline 1 & 161 & 8.7 \\
\hline 3 & 89 & 5.7 \\
\hline 5 & 68 & 5.0 \\
\hline 10 & 48 & 2.5 \\
\hline
\end{tabular}

meters. As the first design step, we computed the size and gain of the patch versus the relative permittivity of the dielectric material. Table 1 shows the result. To a first-order approximation, the patch size is inversely proportional to the square root of the relative permittivity and the gain is proportional to the patch area. When the permittivity of the dielectric material is 10 , the size and gain of the patch is reduced by factors of 3.4 and 4.1 respectively.

The relative permittivity of 10 is usually obtainable only through the use of synthetic ceramic material which is very costly. Among many plastic materials, the monocast(MC) nylon is chosen based on dielectric properties, availability and the cost. The dielectric constant and loss tangent of the MC nylon are measured using the waveguide cell and found to be 2.7 and 0.01 , respectively.

Next we investigate the dependence of the patch resonant frequency on the dielectric height. Fig. 2 shows the result. Reducing the dielectric height lowers the reso-

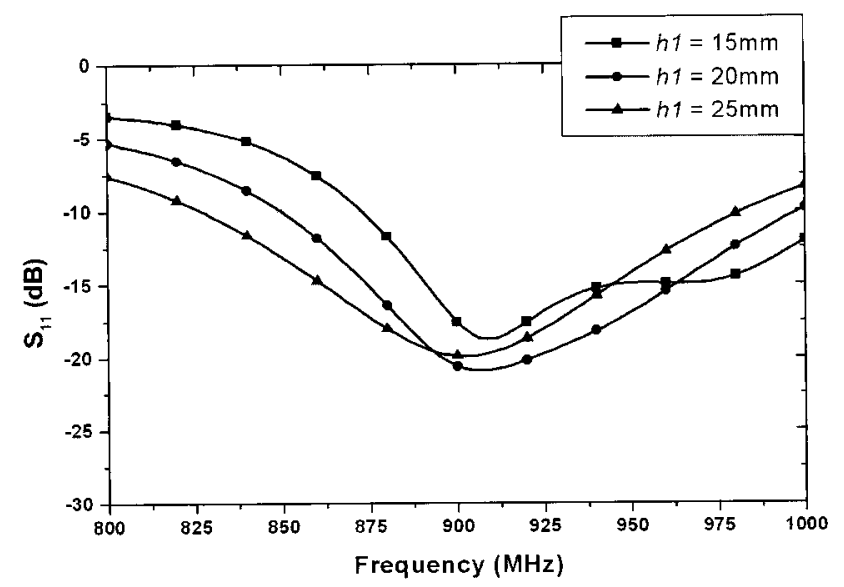

Fig. 2. Effect of the dielectric height on the reflection coefficient of the patch.

$\left(W_{\mathrm{l}}=L_{1}=160, W_{2}=L_{2}=120, W_{3}=L_{3}=93, t=21, s=7, h_{2}=30, \varepsilon_{r}=\right.$ 2.7, unit: $\mathrm{mm}$ ) 


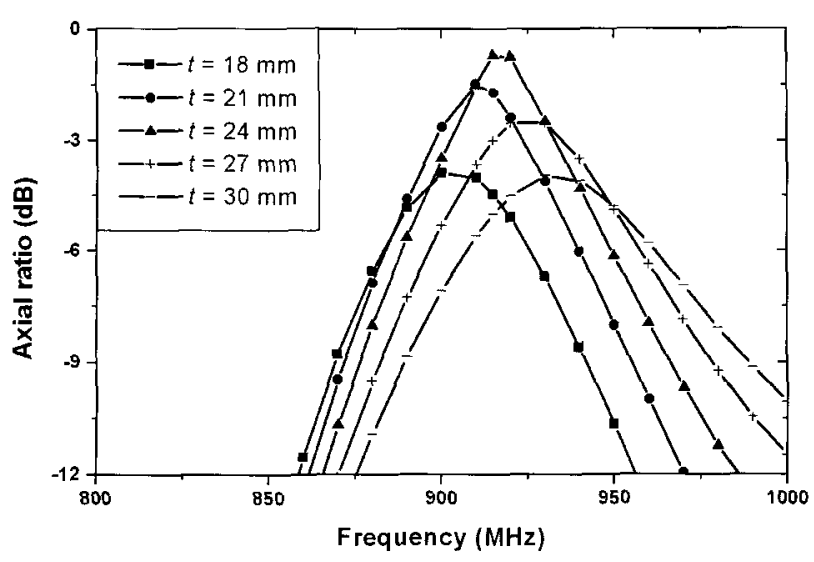

Fig. 3. Dependence of the axial ratio on the patch truncation.

$\left(W_{1}=L_{1}=160, W_{2}=L_{2}=120, W_{3}=L_{3}=93, s=7, h_{1}=20, h_{2}=30, \varepsilon_{r}=\right.$ 2.7, unit: $\mathrm{mm}$ )

nant frequency of the patch because the fringing effect on patch edges is decreased.

Next we investigate the effect of the patch truncation on the antenna performance. Fig. 3 shows the dependence of the axial ratio on the patch truncation. For a given patch length, there is an optimum truncation for the best axial ratio performance. Fig. 4 shows the reflection coefficient of the patch for various values of the truncation. Since the truncation also affects the reflection coefficient of the patch, the axial ratio and the reflection coefficient are simultaneously optimized by iteratively adjusting the patch length and the patch truncation.

The distance $s$ from the patch edge to the vertical ground plane sensitively affects the input impedance of the antenna. Fig. 5 shows the reflection coefficient of

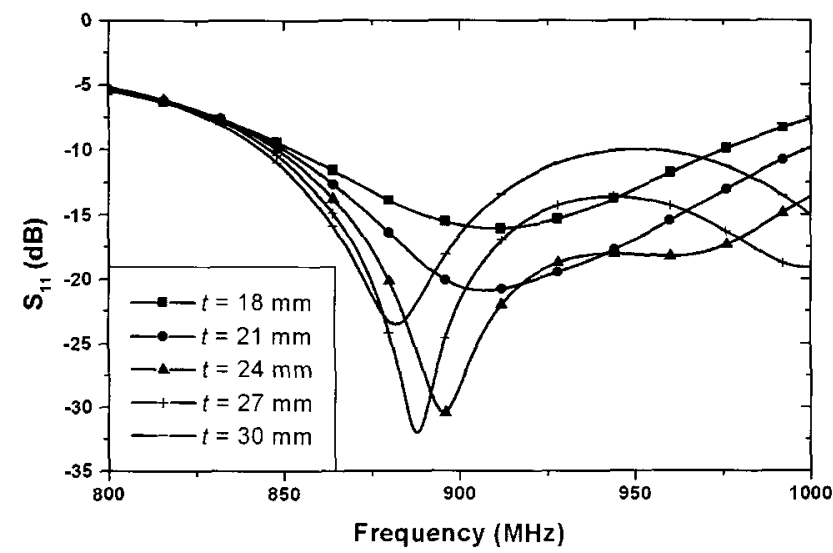

Fig. 4. Reflection coefficient for various values of the patch truncation.

$\left(W_{1}=L_{1}=160, W_{2}=L_{2}=120, W_{3}=L_{3}=93, s=7, h_{1}=20, h_{2}=30, \quad \varepsilon_{r}=\right.$ 2.7, unit: $\mathrm{mm}$ )

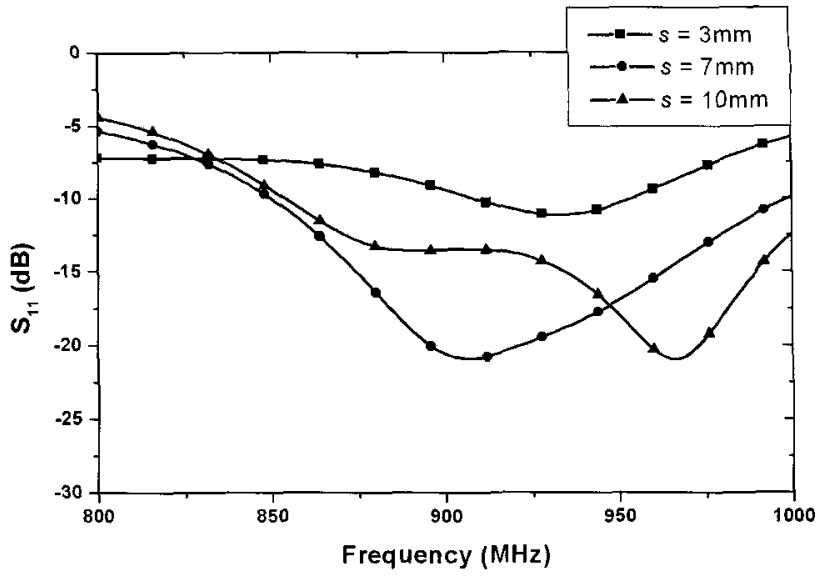

Fig. 5. Dependence of the patch reflection coefficient on the distance from the patch edge to the vertical ground plane.

$\left(W_{1}=L_{1}=160, W_{2}=L_{2}=120, W_{3}=L_{3}=93, t=21, h_{1}=20, h_{2}=30, \varepsilon_{r}=\right.$ 2.7, unit: $\mathrm{mm}$ )

the patch for various values of $s$. The minimum reflection coefficient at $910 \mathrm{MHz}$ occurs when $\mathrm{s}$ is $7 \mathrm{~mm}$.

Finally we investigate the dependence of the antenna performance on the ground plane size. Figs. 6 8 show the result. Fig. 6 shows the gain and back lobe level versus the ground plane size. As the ground plane size is increased, the gain is slightly increased and the back lobe level is significantly decreased. With an $160 \times 160 \mathrm{~mm}$ ground plane, we obtain $5.2-\mathrm{dB}$ gain and $-15-\mathrm{dB}$ back lobe level. Fig. 7 shows the effect of the ground plane size on the patch reflection coefficient. When the ground plane is too small, the reflection coefficient is modified. Fig. 8 shows the dependence of the axial ratio on the ground plane size.

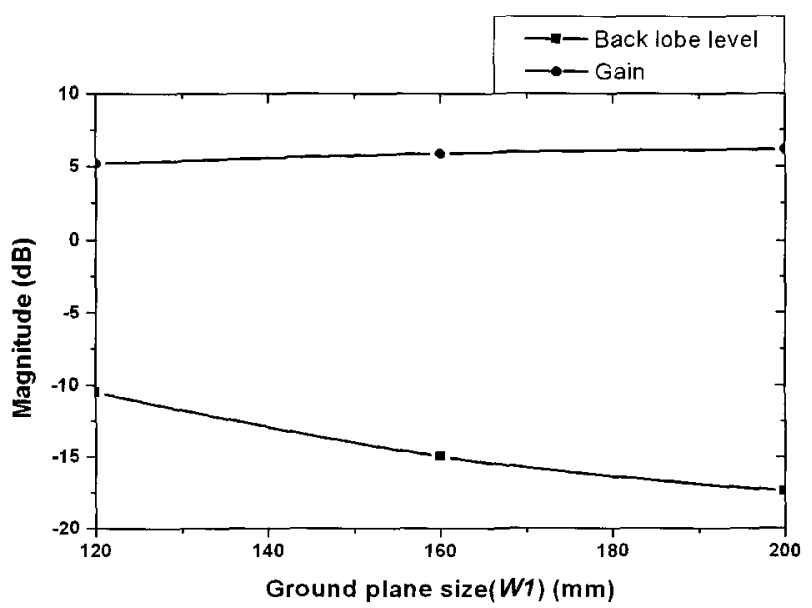

Fig. 6. Gain and back lobe level versus the ground plane size.

$\left(W_{1}=L_{1}, \quad W_{2}=L_{2}=120, \quad W_{3}=L_{3}=93, \quad t=21, \quad h_{1}=20, \quad h_{2}=30, \quad \varepsilon_{r}=\right.$ 2.7 , unit: $\mathrm{mm}$ ) 


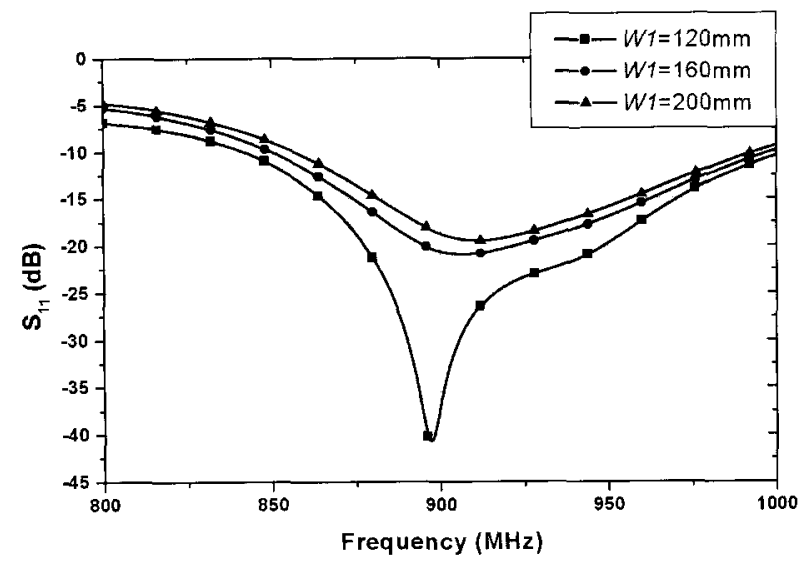

Fig. 7. Effect of the ground plane size on the patch reflection coefficient.

$\left(W_{1}=L_{1}, \quad W_{2}=L_{2}=120, \quad W_{3}=L_{3}=93, \quad t=21, \quad h_{1}=20, \quad h_{2}=30, \quad \varepsilon_{r}=\right.$ 2.7 , unit: $\mathrm{mm}$ )

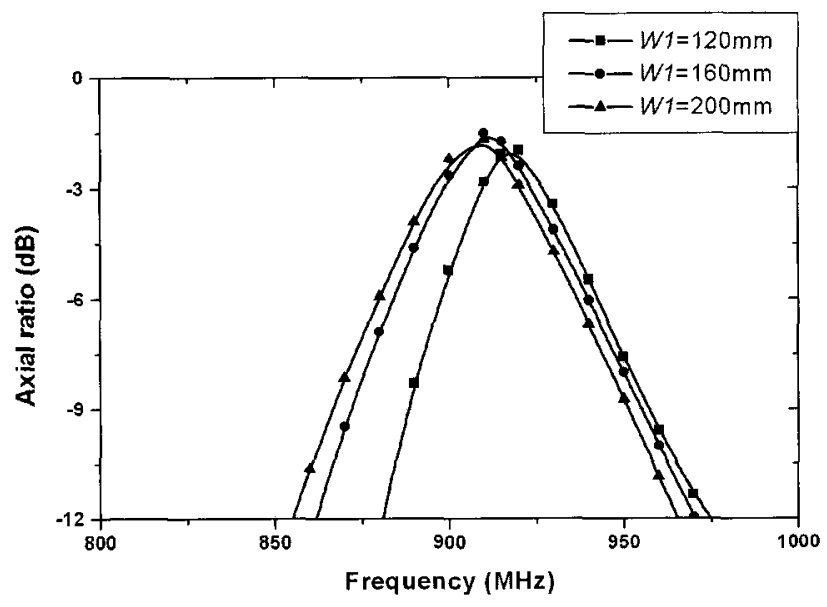

Fig. 8. Effect of the axial ratio on the ground plane size.

$\left(W_{1}=L_{1}, \quad W_{2}=L_{2}=120, \quad W_{3}=L_{3}=93, \quad t=21, \quad h_{1}=20, \quad h_{2}=30, \quad \varepsilon_{r}=\right.$ 2.7, unit: $\mathrm{mm}$ )

The axial ratio, likewise, is affected if the ground plane size is too small.

Based on parametric studies, we arrive at the final antenna design: $W_{1}=L_{1}=160 \mathrm{~mm}, W_{2}=L_{2}=120 \mathrm{~mm}, W_{3}=$ $L_{3}=93 \mathrm{~mm}, t=21 \mathrm{~mm}, h_{1}=20 \mathrm{~mm}, h_{2}=30 \mathrm{~mm}, \varepsilon_{r}=2.7$. With no dielectric loading, the patch size will be $161 \times$ $161 \mathrm{~mm}$. Thus a linear dimensional reduction factor of 1.73 is achieved by loading the patch with mono-cast nylon. The simulated performance of the designed antenna is presented in the next chapter along with the measured performance.

\section{Antenna Fabrication and Measurements}

The designed antenna is fabricated using standard ma-

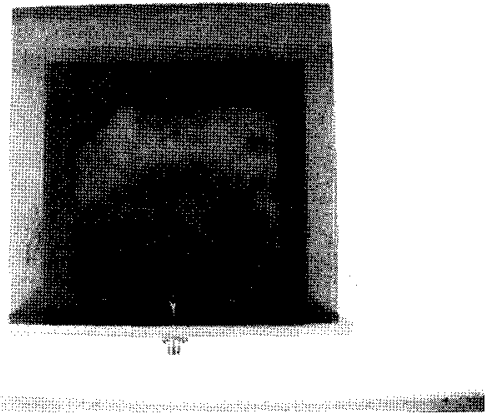

Fig. 9. Photograph of the fabricated antenna.

chining processes. The patch is constructed by cutting a $0.2-\mathrm{mm}$ thick copper plate to accurate size. A probe in the SMA connector is connected to the edge of the patch. A small portion of the probe is placed on top of the patch and soldered to the patch surface. A block of mono-cast nylon is machined into a size of $160 \times 160 \times 20 \mathrm{~mm}$ and placed between the patch and ground plane. The L-shaped ground plane is constructed by joining two $3-\mathrm{mm}$ thick aluminum plates together with bolts. Fig. 9 shows the photograph of the fabricated antenna.

The performance of the fabricated antenna is measured and compared with the simulation. Fig. 10 shows the reflection coefficient of the fabricated antenna. The measured reflection coefficient is below $-10 \mathrm{~dB}$ at 845 $\sim 970 \mathrm{MHz}$. Compared with the simulation, the measured frequency of operation is slightly shifted downwards. Fig. 11 shows the gain of the fabricated antenna. The measured gain of the antenna is $6.0 \mathrm{~dB}$ in close agreement with the simulated value.

Fig. 12 shows the radiation pattern of the fabricated antenna. The vertical and horizontal planes are parallel

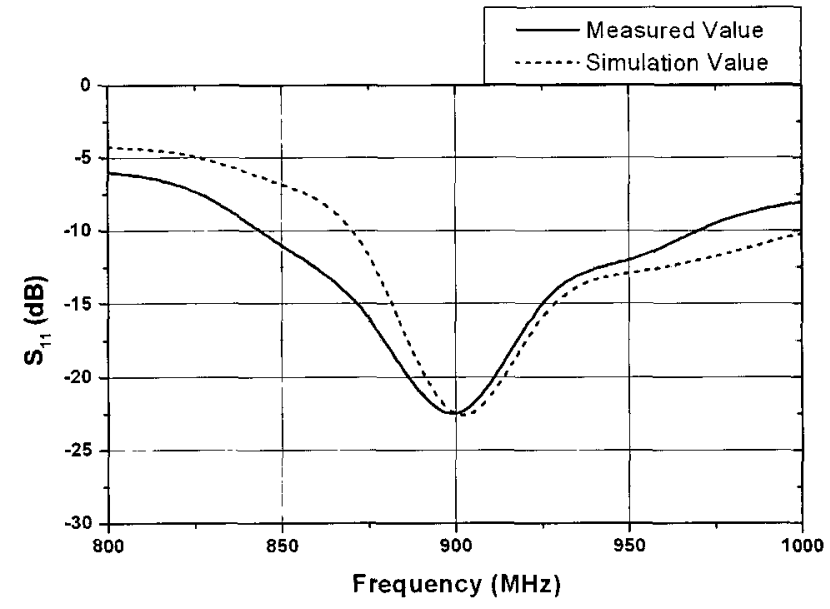

Fig. 10. Reflection coefficient of the fabricated antenna. 


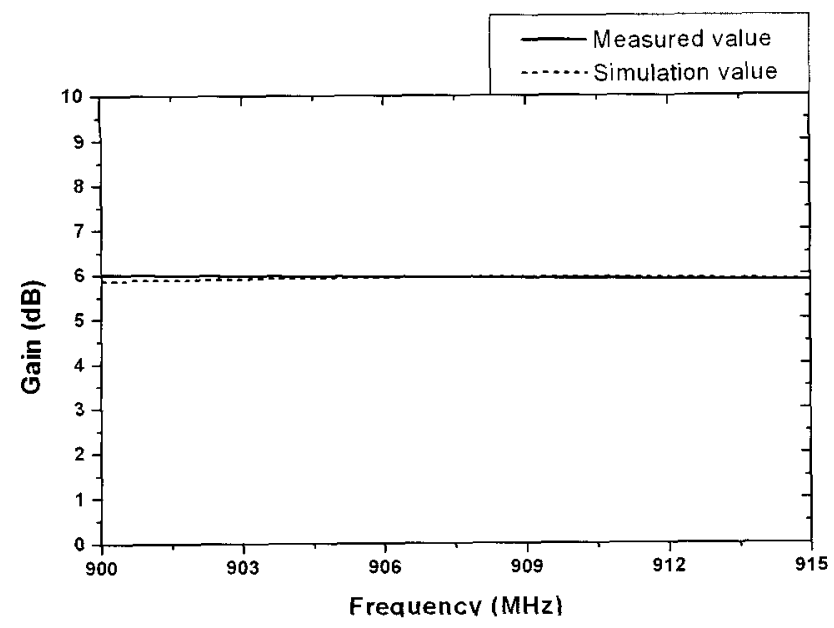

Fig. 11. Gain of the fabricated antenna.

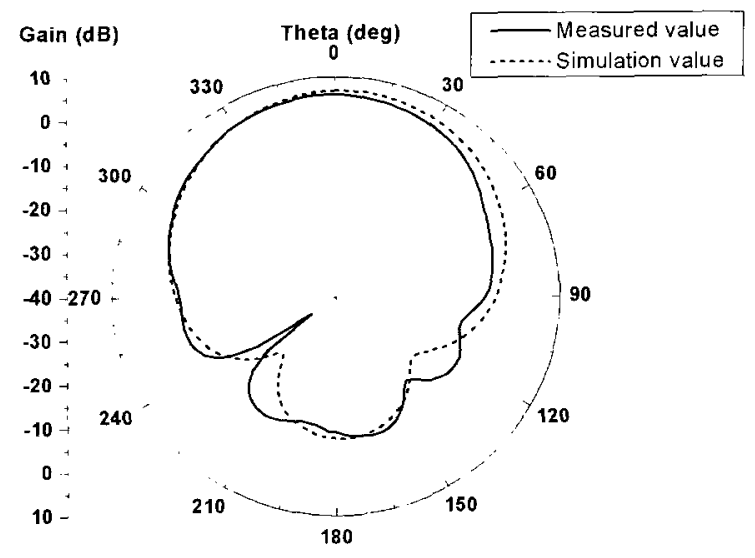

(a)

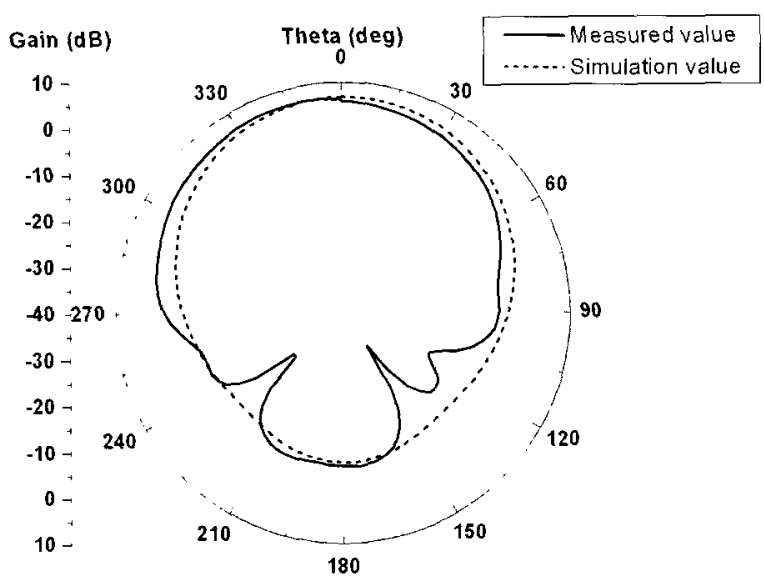

(b)

Fig. 12. Radiation pattern of the antenna at $910 \mathrm{MHz}$ on (a) horizontal plane and (b) vertical plane.

and perpendicular to the probe direction, respectively. Measured 3-dB beamwidths are 87 and 105 degrees on horizontal and vertical planes, respectively. The back lobe level is approximately $-14 \mathrm{~dB}$. We can observe

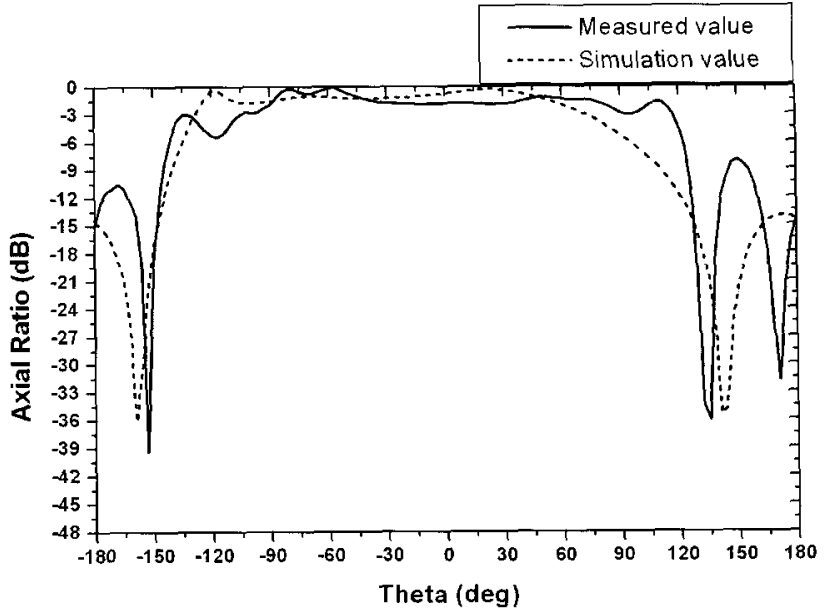

(a)

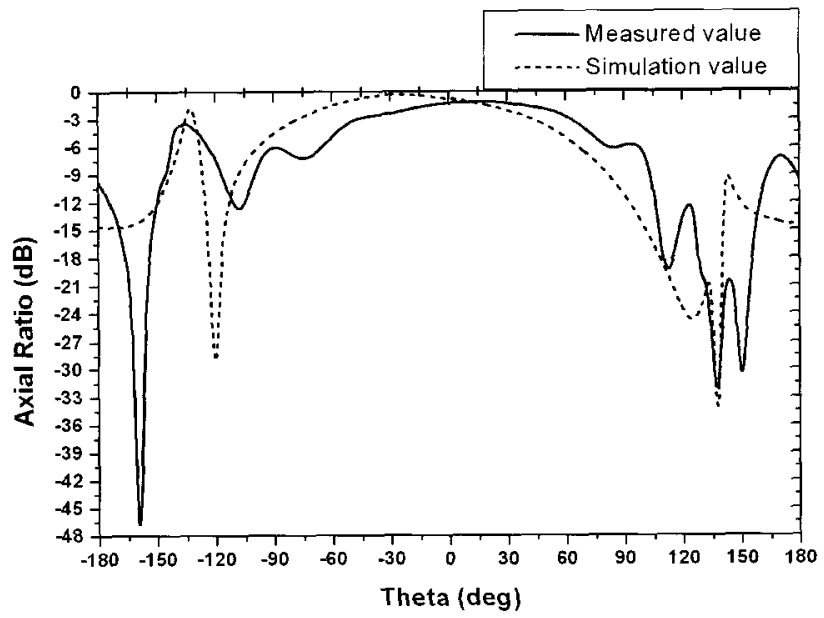

(b)

Fig. 13. Axial ratio pattern of the antenna at $910 \mathrm{MHz}$ on (a) horizontal plane and (b) vertical plane.

good agreements between the measurement and the simulation. Fig. 13 shows the axial ratio pattern of the antenna. For the simplicity in drawing graphs, positive values of the axial ratio are made negative. In Fig. 13 we observe that the fabricated antenna has good axial ratio performances although there are some asymmetries in the angular pattern of the axial ratio. The measured axial ratio is smaller than $3 \mathrm{~dB}$ over 200 and 110 degrees on horizontal and vertical planes, respectively. The agreement of the axial ratio between the measurement and the simulation is fair. The fabricated antenna has an axial ratio less than $3 \mathrm{~dB}$ over $895 \sim 920 \mathrm{MHz}$.

As a check on the field performance of the fabricated antenna, we tested the tag read range. We employed an RFID system by Alien Company with EPC Class 1 UHF RFID tag(64bit) - "Squiggle $T$ ". The fabricated antenna shows a maximum read range of 5 meters, which is same as that of the Alien's 6-dB RFID reader antenna with dimension $260 \times 260 \times 30 \mathrm{~mm}$. 


\section{Conclusions}

In this paper, we present a side-fed dielectric-loaded circularly-polarized patch antenna operating at the 908 $914 \mathrm{MHz}$ frequency band allocated in Korea for RFID applications. The circular polarization is obtained by truncating two diagonal edges of the patch in an appropriate amount. The bandwidth of the patch is greatly enhanced by increasing the distance from the ground plane and the patch. A good impedance property is obtained by feeding the patch on the edge with a coaxial probe via the vertical portion of the L-shaped ground plane. We were able to reduce the patch size by a factor of 1.73 by loading the patch with mono-cast nylon whose dielectric constant is 2.7. Measurements of the fabricated antenna show good performances: $6.0 \mathrm{dBi}$ gain, beamwidths of 87 and 105 degrees on horizontal and vertical planes, reflection coefficient less than -10 $\mathrm{dB}$ over $845 \sim 970 \mathrm{MHz}$, axial ratio less than $3 \mathrm{~dB}$ over 200 and 110 degrees on horizontal and vertical planes, and front back ratio of $-15 \mathrm{~dB}$. The antenna proposed in this paper is small and low-cost, and has the same performance as the standard RFID reader antenna so that it may as well be employed in commercial RFID applications.

\section{References}

[1] K. Sangani, "RFID sees all", IEE Review, vol. 50, no. 4, pp. 22-24, Apr. 2004.

[2] R. Weinstein, "RFID: a technical overview and its application to the enterprise", IT Professional, vol. 7, no. 3, pp. 27-33, May-Jun. 2005.

\section{Ji-Young Jeong}

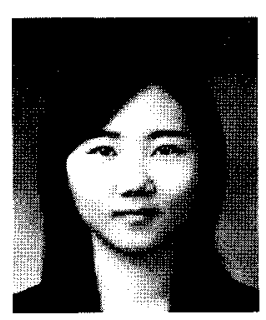

received the B.E. degree in electrical \& computer engineering from Chungbuk University in 2006. She is currently working toward the M.E. degree in radio engineering department, Graduate School, Chungbuk University. Her research interests include antennas and microwave circuits.
[3] Korean RFID/USN, http://www.karus.or.kr/

[4] J.-Y. Choo, H.-S. Choo, I.-M. Park, and Y.-S. Oh, "Design of crooked wire antennas for UHF band RFID reader", J. Korea Electromag. Eng. Soc., vol. 16 , no. 5, pp. 472-481, May 2005.

[5] P. Raumonen, M. Keskilammi, L. Sydanheimo, and M. Kivikoski, "A very low profile CP EBG antenna for RFID reader", Dig. IEEE 2004 Antennas Propagat. Soc. Int. Symp., pp. 3808-3811, Jun. 2004.

[6] X. Qing, N. Yang, "2.45 GHz circularly polarized RFID reader antenna", Dig. IEEE 9th Int. Conf. Comm. Sys. (ICCS 2004), pp. 612-615, Sep. 2004.

[7] S. K. Pahdi, N. C. Karmakar, and C. L. Law, "An EM-coupled dual-polarized microstrip patch antenna for RFID applications", Microwave Opt. Tech. Lett., vol. 39, no. 5, pp. 354-360, Dec. 2003.

[8] M. Keskilammi, L. Sydanheimo, and M. Kivikoski, "Radio frequency technology for automated manufacturing and logistics contorl. Part I : Passive RFID systems and the effects of antenna parameters on operational distance", Int. J. Adv. Manuf. Technol., vol. 21, no. 10-11, pp. 769-774, Jul. 2003.

[9] F.-S. Chang, K.-L. Wong, and T.-W. Chiou, "Lowcost broadband circularly polarized patch antenna", IEEE Trans. Antennas Propagat., vol. 51, no. 10, pp. 3006-3009, Oct. 2003.

[10] M. Niroojazi, M. N. Azarmanesh "Practical design of single feed truncated corner microstrip antenna", Proc. IEEE 2nd Annual Conf. on Communication Networks and Services Research, pp. 25-29, May 2004.

\section{Seung-Mo Choi}

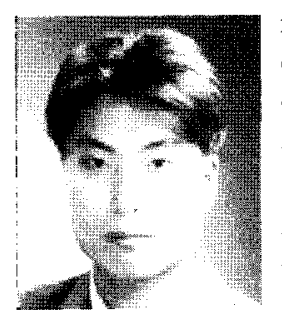

received the B.E. degree in electrical \& computer engineering in 2004 and M.E. degree in radio engineering department in 2006, both from Chungbuk University. $\mathrm{He}$ is currently working at Mito-RF Company. His research interests include antennas and microwave circuits. 


\section{Bayanmunkh Enkhbayar}

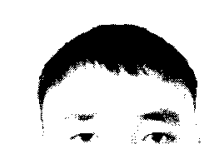

received the B.E. and M.E. degrees in telecommunication engineering from Mongolian University of Since and Technology in 2005 and 2006, respectively. He is currently working toward the Ph.D. de- gree in radio engineering department, Gra-

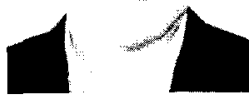
duate School, Chungbuk University. His research interests include antennas and microwave circuits.

\section{Ononchimeg Sodnomtseren}

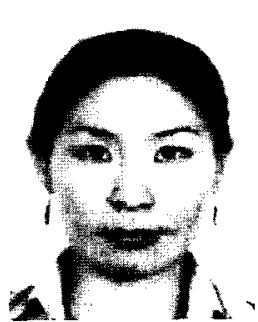

received the B.E. and M.E. degrees in radio communications engineering from Mongolian University of Science and Technology in 2002 and 2003, respectively. She is currently working toward the Ph.D. degree in Radio Engineering Department, Graduate School, Chungbuk University. Her research interests include antennas and microwave circuits.

\section{Bierng-Chearl Ahn}

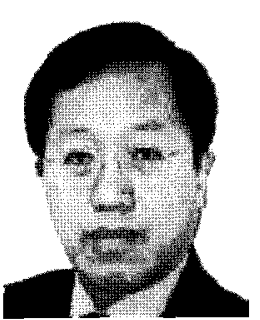

received the B.E. degree in electrical engineering from Seoul National University in 1981, the M.E. degree in electrical engineering form Korea Advanced Institute of Science and Technology in 1983, and the Ph.D. degree in electrical engineering from University of Mississippi in 1992. From 1983 to 1986, he worked as a research engineer at Goldstar Precision Company. From 1993 to 1994, he was with Agency for Defense Development. Since 1995 he has been with Chungbuk University, where he is currently a professor in the school of electrical and computer engineering. His research interests include applied electromagnetics and antennas. 